\title{
Resultados y complicaciones de la técnica TVT en el tratamiento de la incontinencia de esfuerzo femenina
}

\author{
D. Cañís Sánchez, Ó. Bielsa Galí, R. Cortadellas Ángel, O. Arango Toro, J. Placer Santos, \\ A. Gelabert-Mas
}

\author{
Servicio y Cátedra de Urología. Hospital del Mar. UAB. Barcelona.
}

Actas Urol Esp 2005; 29 (3): 287-291

\section{RESUMEN \\ RESULTADOS Y COMPLICACIONES DE LA TÉCNICA TVT EN EL TRATAMIENTO DE LA INCONTINENCIA DE ESFUERZO FEMENINA}

Objetivos: Evaluar la eficacia y seguridad de la cinta vaginal sin tensión (TVT) en el tratamiento de la incontinencia urinaria de esfuerzo femenina (IUE).

Analizar los resultados a medio plazo de la asociación de la TVT con la corrección quirúrgica de prolapsos genitales.

Pacientes y método: Se estudian 100 pacientes con IUE intervenidas de forma consecutiva en nuestro centro bajo anestesia regional. A todas ellas se les implantó la TVT y en 24 casos además, se corrigió un cistocele grado II-III. Fue necesario en 3 de estas 24 pacientes reparar un rectocele y se practicaron 4 histerectomías vaginales en el mismo acto quirúrgico.

Resultados: La edad media de las mujeres fue de 49,3 años (35-78), el número promedio de partos de 2 (0-6), la duración media de la intervención de 38 minutos (20-50) y todas las pacientes a las que se colocó la TVT sin otras correcciones quirúrgicas obtuvieron el alta hospitalaria a las 24 horas de la intervención. Tan sólo una paciente precisó de sonda varios días por retención urinaria.

El tiempo medio de seguimiento fue de 18 meses (12-48) y la IUE se solucionó en el 95\% de los casos. Como complicación más frecuente observamos la aparición de hiperactividad detrusorial sintomática "de novo" en el 16\% de la serie; la perforación vesical en una de las pacientes fue el problema más grave.

Conclusiones: La TVT es una técnica sencilla, segura, eficaz y relativamente económica para el tratamiento de la IUE.

La TVT puede asociarse con éxito a la corrección quirúrgica de prolapsos genitales. Este hecho prolonga la estancia hospitalaria en 48 horas sin incrementar la morbilidad de la cirugía.

Palabras clave: Incontinencia de esfuerzo. TVT. Prolapsos genitales.

\footnotetext{
ABSTRACT

RESULTS AND COMPLICATIONS OF TVT PROCEDURE FOR SURGICAL TREATMENT OF FEMALE STRESS INCONTINENCE

Objetives: To evaluate the efficacy and safety of Tension-free vaginal tape (TVT) for treatment of female stress urinary incontinence (SUI).

To determine the mid-term outcome of TVT performed during other pelvic floor reconstructive procedures.

Patients and methods: 100 women with SUI undergoing TVT procedure under spinal anesthesia from January 2000 to November 2002 were studied.

76 women were treated with TVT alone. 24 patients were treated with TVT and pelvic floor reconstruction: we repaired 24 cystoceles grade II-III, 3 rectoceles and 4 concomitant vaginal histerectomies.

Results: Mean age was 49,3 years (range 35-78). Mean parity: 2 (range 0-6) and mean operative time was 38 minutes (range 20 to 50). The postoperative hospital stay was 24 hours for women treated with TVT alone. Only 1 patient ( $1 \%)$ need bladder catheterization during 7 days due to urinary retention.

Mean followup was 18 months (range 12-48). Objetive cure rate was 95\%. In our study the rate of de novo post-operative urge symptoms $(16 \%)$ was the most frequent complication. Bladder injury was the most grave problem and it happened in 1 patient (1\%).

Conclusion: The results confirm the feasibility and safety of TVT for treatment of SUI. Moreover, TVT procedure is economical and efectiveness.

Pelvic floor defects, benign uterine disorders and SUI can be safely treated with TVT and vaginal procedures during the same surgical time.
}

Keywords: Stress incontinence. TVT. Genital prolapses. 
$\mathrm{E}$ xisten en la literatura médica actual más de 100 procedimientos quirúrgicos descritos para el tratamiento de la incontinencia de esfuerzo (IUE). En las dos últimas décadas hemos asistido al ascenso y al declive de muchos de ellos.

Hasta hace relativamente poco tiempo la colposuspensión tipo Burch seguía siendo el "gold estandar" para la mayoría de urólogos. Con esta técnica clásica se consiguen tasas de curación objetiva del 80\% al 90\% a los 3 años de seguimiento ${ }^{1}$. Incluso a más largo plazo, estas cifras se mantienen por encima del $80 \%{ }^{2}$. Sin embargo el acceso retropúbico tradicional requiere una incisión abdominal y maniobras de disección que pueden asociarse a una morbilidad significativa durante la cirugía y después de ella (disfunción miccional, prolapso útero-vaginal) así como prolongadas estancias hospitalarias que se traducen en un elevado coste económico.

Cuando Ulmsten y sus colaboradores describieron la TVT por primera vez en 1996 estaba bien fundamentada y había sido precedida de años de investigación experimental. El objetivo era lograr un procedimiento sencillo, mínimamente invasivo y relativamente económico para corregir la $\mathrm{IUE}^{3}$.

La técnica se basa en la restauración de los ligamentos pubo-uretrales mediante el implante de un soporte suburetral de polipropileno en uretra media, sin tensión y a través de una minima incisión vaginal.

Desde aquel primer artículo la experiencia internacional ha verificado de forma notablemente uniforme las tasas de éxito (subjetivo y objetivo) y de complicaciones, por lo que esta técnica ha ido generalizándose con rapidez.

En la actualidad, la TVT compite claramente con la colposuspensión clásica también en cuanto a su eficacia a largo plazo:

Según las series de Nilsson y Rezapour, las tasas de curación objetiva con un tiempo de seguimiento superior a los 3 años superan el 85\% en pacientes con IUE genuina y también con incontinencia mixta ${ }^{4,5}$.

Rezapour y Falconer reportan en el año 2001 un $74 \%$ de éxitos a largo plazo en pacientes con insuficiencia esfinteriana intrínseca (DEI) a las que se implantó la TVT $^{6}$.
Nuestro trabajo pretende analizar la eficacia y seguridad a medio plazo de la TVT en las 100 primeras pacientes intervenidas en un hospital de tercer nivel. Presentamos así mismo los resultados obtenidos en la asociación de la cinta vaginal sin tensión a la correción de distintos prolapsos genitales en el mismo tiempo quirúrgico.

\section{PACIENTES Y MÉTODO}

Se estudiaron las 100 primeras pacientes intervenidas mediante técnica de TVT en nuestro centro entre enero del año 2000 y noviembre del 2002. Todas ellas estaban diagnosticadas de IUE; 15 mujeres (15\%) presentaban además sintomatología de urgencia miccional y 3 (3\%) fueron diagnosticadas de insuficiencia esfinteriana intrínseca (con presiones de cierre uretral <20 $\mathrm{cmH}_{2} \mathrm{O}$ ). La edad media de las pacientes fue de 49,3 (35-78) años y la paridad de 2 (0-6).

En 6 de estas pacientes (6\%) la IUE era recidivante, habiendo sido sometidas previamente a alguna intervención pélvica: identificamos 3 reparaciones anteriores con plastia de Kelly, 2 procedimientos de Marshall-Marchetti-Krantz y 1 colposuspensión de Burch.

Todas las pacientes fueron sometidas al procedimiento de TVT bajo anestesia regional. Se hizo una incisión sagital de $1,5 \mathrm{~cm}$ de longitud a nivel de la pared vaginal anterior. Tras una mínima disección parauretral se introdujo una cinta especial de polipropileno (Prolene) recubierta de una funda plástica (Ethicon Inc, Sommerville, New Jersey, USA) utilizando dos agujas. La punta de estas agujas, primero perfora el diafragma urogenital y luego se mueve hacia arriba dentro del espacio retropúbico. Tras la perforación de la vaina del recto, se hace una pequeña incisión de $1 \mathrm{~cm}$ sobre la punta de la aguja, justo tras el borde superior del pubis. Esta maniobra se repite entonces en el otro lado con la otra aguja asegurándose de que las incisiones de la piel quedan separadas por unos $5 \mathrm{~cm}$ de distancia.

Con las agujas aún insertadas se realizó una uretrocistoscopia para asegurar que la vejiga no había sufrido ningún daño. Las agujas se sacan a través de las incisiones suprapúbicas y la cinta se ajusta sin tensión bajo el tercio medio de uretra. Llegados a este punto se retira la vaina plástica que recubre la cinta de Prolene, la cual no 
requiere fijación alguna. La técnica concluye con el cierre de las incisiones cutáneas y de la incisión vaginal. La uretra se comprueba con un dilatador de Hegar. Se coloca una sonda Foley $18 \mathrm{Ch}$ y un taponamiento vaginal que se retirarán a las 24 horas.

Se utilizó una dosis única de antibiótico profiláctico intraoperatorio con Amoxicilina-clavulánico. Se registró sistemáticamente, la duración de la operación, la cantidad de sangre contenida en el aspirador, cualquier incidencia intraoperatoria y se solicitó un hemograma de control a las 24 horas.

$24(24 \%)$ de las pacientes necesitaron diversos procedimientos de reconstrucción de prolapso vaginal además de la TVT:

Se repararon 24 cistoceles grado II ó III; se corrigieron 3 rectoceles y se practicaron 4 histerectomías vaginales. Los 2 cirujanos que participaron en estas intervenciones fueron Urólogos con una amplia experiencia en esta vía de abordaje.

Las 100 pacientes fueron incluidas en un protocolo de estudio que consistía en un diario miccional durante 2 días, una exploración física (prueba de provocación por la tos) en decúbito y en bipedestación, un urinocultivo, un estudio urodinámico (flujometría, presión-flujo y perfil uretral) previo a la intervención y una uretrocistografía en aquellas pacientes que referían antecedentes quirúrgicos previos.

Tras la intervención quirúrgica se repetía el diario miccional, la exploración física y se determinaba ecográficamente el residuo postmiccional.

Después de la cirugía consideramos que las pacientes estaban curadas si presentaban una prueba de provocación por la tos negativa y una reducción del $75 \%$ en el número de compresas diarias y en la calidad de vida, evaluada mediante una escala analógica visual en la que el 0 representaba la ausencia de problemas urinarios y el 100 la presencia de molestias insoportables.

Como ha quedado expuesto, no fueron criterios de exclusión del estudio ni por lo tanto, contraindicación para la cirugia: la edad, la existencia de clínica de urgencia, la hiperactividad detrusorial demostrada que responde favorablemente al tratamiento con anticolinérgicos, la deficiencia esfinteriana intrínseca, la IUE recurrente tras cirugías pélvicas previas ni la coexistencia de prolapso uterino.

\section{RESULTADOS}

El tiempo de seguimiento medio fue de 18 meses (intervalo: 12-48) siendo posible evaluar según el protocolo a la totalidad de las pacientes. La duración media de la intervención quirúrgica en aquellas mujeres a las que se les sometió exclusivamente a la TVT fue de 38 (20-50) minutos. La estancia hospitalaria media para estas 76 pacientes fue de 2 días, teniendo en cuenta que ingresaron la noche previa a la intervención y obtuvieron el alta hospitalaria al día siguiente. La sonda vesical y el taponamiento vaginal se retiraron a las 24 horas de la cirugía. Aquellas pacientes a las que se les practicó la corrección quirúrgica de algún tipo de prolapso genital permanecieron en el hospital durante 4 días.

La pérdida estimada de sangre fue en todos los casos inferior a los $200 \mathrm{ml}$ y el descenso postoperatorio de la Hemoglobina fue en promedio de $0,4(0,2-1,1)$ puntos. No fue necesario por lo tanto, transfundir a ninguna paciente.

La intervención dio lugar a pocas complicaciones. En 1 caso (1\%) se observó un hematoma clínico de los músculos rectos del abdomen. Una paciente $(1 \%)$ hizo una retención urinaria que precisó de sondaje durante 7 días, tras los cuales consiguió micciones confortables con residuos inferiores a los $60 \mathrm{ml}$. Dos (2\%) mujeres presentaron cierto grado de disuria durante los 4 primeros días postintervención. Durante los 2 primeros meses de seguimiento diagnosticamos 3 (3\%) infecciones urinarias bajas (ITU) que respondieron favorablemente al tratamiento antibiótico. 2 de estas pacientes habían sido portadoras de sonda urinaria durante 36 horas porque se les corrigió un cistocele durante la intervención.

El problema más grave ocurrido fue la perforación vesical en una (1\%) de las mujeres. Éste se manejó mediante la retirada y el reposicionamiento de la aguja y con el sondaje vesical durante 3 días.

El hallazgo de hiperactividad detrusorial sintomática "de novo" en 16 (16\%) mujeres aparece como la complicación más frecuente en nuestra serie y la prescripción de fármacos anticolinérgicos en 14 de estas pacientes consiguió una curación objetiva y también subjetiva.

Observamos en $3(3 \%)$ pacientes la aparición de dolor en uno de los orificios de salida de las 
agujas que, en 1 caso se prolongó hasta los 3 meses de seguimiento y sin que la exploración sugiriera la existencia de una osteitis de pubis. En una paciente se diagnosticó una extrusión vaginal de la cinta que se solucionó con un colgajo de pared vaginal realizado bajo anestesia local. Ninguna mujer desarrolló infección de la cinta ni de la herida quirúrgica. Hasta la fecha no reportamos ningún caso de infección urinaria recurrente. Ninguna paciente presenta sintomatología obstructiva importante y los residuos postmiccionales detectados en 4 (4\%) pacientes son inferiores a los $60 \mathrm{ml}$.

Tras la aplicación de la técnica, 95 (95\%) pacientes quedaron curadas según los criterios de curación ya descritos, es decir, obtuvimos una tasa de curación objetiva a medio plazo del 95\%. Las 5 pacientes restantes mejoraron de forma significativa y aunque esta mejoría fue también subjetiva, fueron consideradas como fracasos.

\section{DISCUSIÓN}

Los resultados de este estudio de seguimiento coinciden con otros trabajos ${ }^{7-10}$. La tasa de curación fue alta (del 95\%) y en coincidencia con las tasas esperadas de la colposuspensión ${ }^{2,11}$. Además, las ventajas aparentes a corto plazo son obvias: se trata de una técnica quirúrgica realizada en menos de 50 minutos y que permite el alta domiciliaria de las pacientes a las 24 horas; el 99\% de éstas consiguieron micción espontánea al retirar el sondaje vesical.

Este elevado porcentaje de micción espontánea inmediatamente después de la intervención es muy prometedor y coincide de nuevo con la experiencia de otros autores ${ }^{12,13}$. Las bajas tasas de retención urinaria (1\%) reflejan probablemente la disección mínima de los tejidos y la posición holgada de la cinta alrededor de la uretra media. En el seguimiento, ninguna mujer presenta sintomatologia urinaria obstructiva importante. El mecanismo propuesto por el que la TVT consigue restaurar la continencia es todavía debatido pero se piensa que podría corregir la existencia de unos ligamentos pubouretrales deficientes, creando una resistencia uretral dinámica bajo el estrés pero que no interfiere la función uretral en reposo. Esta teoría puede explicar en gran parte la baja incidencia de complicaciones miccionales.
Las complicaciones intraoperatorias del procedimiento se asocian fundamentalmente con el riesgo de perforación vesical. En nuestra serie, tan sólo 1 ( $1 \%)$ mujer sufrió perforación. Los porcentajes publicados en la literatura oscilan entre el 0-9\% $\%^{14,15}$ y siempre que esta complicación sea detectada, no parece tener implicaciones a medio y largo plazo. Las lesiones potencialmente más graves son las del nervio obturador y especialmente la de los vasos ilíacos. Aunque ambas se han descrito en algunos trabajos ${ }^{16,17}$, son complicaciones raras $\mathrm{y}$ no se han presentado en nuestra serie.

En el análisis de las complicaciones diferidas, observamos tan solo $3(3 \%)$ casos de ITU. Este hecho traduce quizás, la ausencia de sondajes prolongados. Sin embargo, la hiperactividad detrusorial sintomática "de novo" se registró como el problema asociado a la cirugía más frecuentemente observado en nuestro centro $(16 \%)^{18}$. Curiosamente, 10 de estas mujeres tuvieron como síntoma una incontinencia de esfuerzo a minimos esfuerzos antes de la cirugía. Las 6 restantes presentaban una clinica compatible con una incontinencia mixta. Es posible por tanto, que esta hiperactividad detrusorial estuviera ya presente y no se detectara en el reconocimiento inicial, o bien sea secundaria a la colocación de la TVT.

El seguimiento a medio plazo demuestra que la TVT es una técnica segura y efectiva para el tratamiento de la IUE genuina y según nuestra experiencia, también lo es para la incontinencia mixta y la DEI. Además, resulta un procedimiento económico que permite una rápida incorporación de las pacientes a las actividades de la vida cotidiana. A pesar de la existencia de múltiples series de seguimiento a largo plazo, esperamos todavía resultados de estudios prospectivos aleatorizados que comparen la TVT con la colposuspensión ${ }^{19,20}$.

Rezapour y Ulmsten publicaron en el año 2001 una tasa de curación del 82\% tras implantar la TVT a 36 pacientes con IUE recidivante y con un seguimiento medio de 4 años. Aunque cabría esperar que este grupo de mujeres resultara difícil de curar por métodos quirúrgicos, los antecedentes de cirugías pélvicas previas no contraindicaron la técnica TVT en nuestro centro. 
Ninguna de las 6 pacientes intervenidas bajo estas circunstancias presentó complicaciones intraoperatorias significativas aunque una de ellas fue la única de la serie que hizo rentención urinaria cuando se retiró el sondaje a las 24 horas. A pesar de que ninguna de estas pacientes ha sido estudiada por un tiempo superior a los 20 meses, no detectamos una disminución de los resultados durante los controles ambulatorios.

En conclusión y a pesar de que el número de pacientes es limitado, nuestros resultados coinciden en gran parte con los de otros autores, por lo que en nuestro Servicio se recomienda la TVT como primera opción terapéutica en la mayoría de mujeres con IUE tributarias de tratamiento quirúrgico. La indicación no se modifica en aquellas pacientes en las que han fracasado otras técnicas tradicionales ó en aquellas que requieren disecciones más complejas por la necesidad de reparar prolapsos genitales asociados.

\section{CONCLUSIONES}

La cinta vaginal sin tensión es a medio plazo, una técnica sencilla, segura, eficaz y relativamente económica para el tratamiento quirúrgico de la incontinencia de esfuerzo.

La TVT puede asociarse con éxito a otras maniobras quirúrgicas para la solución de prolapsos genitales. Este hecho prolonga la estancia hospitalaria en 48 horas sin incrementar la morbilidad de la cirugía.

\section{REFERENCIAS}

1. Ulmsten U, Falconer C, Johnson P, et al. A multicenter study of TVT (tension free vaginal tape) for surgical treatment of stress urinary incontinence. Int Urogynecol $\mathrm{J}$ 1998;9:210-213.

2. Alcalay M, Monga A, Stanton SL. Burch colposuspension: a 1020 year follow-up. Br J Obstet Gynaecol 1995; 102:740-745.

3. Ulmsten U, Henriksson L, Johnson P, Varhos G. An ambulatory surgical procedure under local anesthesia for treatment of female urinary incontinence. Int Urogynecol $\mathrm{J}$ 1996;7:81-86.

4. Nilsson CG, Kuuva N, Falconer C, Rezapour M, Ulmsten U. Long-term results of the tension-free vaginal tape (TVT) procedure for surgical treatment of female stress urinary incontinence. Int Urogynecol J 2001;Suppl12(4):65-68.

5. Rezapour M, Ulmsten U. Tension-free vaginal tape (TVT) in women with mixed urinary incontinence-a long term followup. Int Urogynecol J Pelvic Floor Dysfunct 2001;12Supp12: S15-18.

6. Rezapour M, Falconer C, Ulmsten U. Tension-Free vaginal tape TVT) in stress incontinent women with intrinsic sphincter deficiency (ISD)-a long-term follow-up. Int Urogynecol $\mathrm{J}$ Pelvic Floor Dysfunct 2001;12 Suppl 2:S12-14.
7. Moran PA, Ward KL, Johnson D, Smirni WE, Hilton P, Bibby J. Tension-free vaginal tape for primary genuine stress incontinence: a two-center follow-up study. BJU Int 2000;86:39-42.

8. Gutierrez Baños JL, Martín García B, Portillo Martín B, Del Valle Schaan JI, Hernández Rodríguez R, Correas Gómez MA, et al. Tratamiento de la incontinencia urinaria de esfuerzo con TVT. 3 años de experiencia. Urod a 2003 16(1): 16-22.

9. Escribano Patino G, Hernández Hernández C, Subirá Ríos D, Castaño González I, Moralejo Garate M, Martínez Salamanca JI.: Incontinencia urinaria femenina de esfuerzo. Tratamiento quirúrgico mediante técnicas de sling pubovaginal. Arch Esp Urol 2002Nov;55(9):1093-1105.

10. Briones Mardones G, Jiménez Cidre M, Fernández Fernéndez E, Perales Cabanas L, Pozo Mengual B, Sanz Miguelanez JL, et al. Tratamiento de la incontinencia urinaria de esfuerzo femenina por la técnica TVT (tension-free vaginal tape): nuestra experiencia. Actas Urol Esp 2001 Jun;25(6):423-429.

11. Feyereisl J, Dreher E, Haenggi W, et al. Long term results after Burch colposuspension. Am J Obstet Gynecol 1994; 171:647-652.

12. Volkmer BG, Nesslauer T, Rinnab L, et al. Surgical intervention for complications of the tension free vaginal tape procedure. J Urol 2002;169:570-574.

13. Rackley RR, Abdelmalak JB, Tchetgen MB, et al. Tensionfree vaginal tape and percutaneous vaginal tape sling procedures. Tech Urol 2001;7:90.

14. Lebret T, Lugagne PM, Herve JM, et al. : Evaluation of tension-free vaginal tape procedure. Its safety and efficacy in the treatment of female stress urinary incontinence during the learning phase. Eur Urol 2001;40:543.

15. Jomaa M. Combined tension-free vaginal tape and prolapse repair under local anaesthesia in patients with symptoms of both urinary incontinence and prolapse. Gynecol Obstet Invest 2001;51:184.

16. Meschia M, Pifarotti P, Bernasconi F, et al. Tension-free vaginal tape: analysis of outcomes and complications in 404 stress incontinent women. Int Urogynecol J Pelvic Floor Dysfunct, 2001;Suppl12:S24.

17. Boustead GB. The tension-free vaginal tape for treating female stress urinary incontinence. BJU Int 2002;89:687693.

18. Rafii A, Paoletti X, Haab F, et al. Tension-free vaginal tape and associate procedures : A case control study. Eur Urol 2004;45:356-361.

19. Ward K, Hilton P. United Kingdom and Ireland Tensionfree Vaginal Tape Trial Group. Prospective multicentre randomised trial of tension-free vaginal tape and colposuspension as primary treatment for stress incontinence. Br Med J 2002;67:325.

20. Liapis A, Bakas P, Creatsas G. Burch colposuspension and tension-free vaginal tape in the management of stress urinary incontinence in women. Eur Urol 2002;41:469-473.

Dr. D. Cañís Sánchez

Servicio de Urología. Hospital del Mar.

Passeig Marítim 25-29

08003 Barcelona

(Trabajo recibido el 10 junio de 2004) 